\title{
Dynamical scaling and intermittency in shell models of turbulence
}

\author{
Roberto Benzi ${ }^{1}$, Luca Biferale ${ }^{1}$ and Mauro Sbragaglia ${ }^{1}$ \\ 1 Dipartimento di Fisica and INFN, Università "Tor Vergata", \\ Via della Ricerca Scientifica 1, I-00133 Roma, Italy.
}

\begin{abstract}
We introduce a model for the turbulent energy cascade aimed at studying the effect of dynamical scaling on intermittency. In particular, we show that by slowing down the energy transfer mechanism for fixed energy flux, intermittency decreases and eventually disappears. This result supports the conjecture that intermittency can be observed only if energy is flowing towards faster and faster scales of motion.
\end{abstract}

Turbulent flows show very different behaviour at changing the embedding physical dimension. The most spectacular change is in the reversal of the energy flux, from large to small scales in $3 d$ and viceversa in $2 d$ [1]. Moreover, the three-dimensional forward energy flux is strongly intermittent while the two-dimensional inverse energy transfer is almost Gaussian [2]. The presence of strong anomalous fluctuations in the $3 d$ flux is believed to be connected to the existence of a hierarchical organization in the eddy turn over times, $\tau_{r}$, at different scales. Simple dimensional arguments predict the eddy turn over time of velocity fluctuations at scale $r$ in the inertial range to be of the order of $\tau_{r} \sim r^{2 / 3}$. For three dimensional turbulence, the scenario is the one of an energy cascade from slow (large eddies) to fast (small eddies), without the possibility for fluctuations at different scales to equilibrate. This mechanism should be at the origin of the burst-like structure in the forward energy transfer mechanism: any unusual fluctuation in the energy content at a given scale propagates to smaller and smaller scales until it is re-adsorbed by viscous mechanism. Hence, this is the cause of strong inhomogeneity in the spatio-temporal statistical properties of the energy transfer process. The same qualitative arguments capture the absence of intermittency in the inverse $2 d$ process, where energy flows from fast to slow modes, allowing the receiving modes to feel only the mean fluctuations of the unstable "mother" eddy. Intermittency in the related problem of scalar passive/active quantities advected by turbulent flows has been connected to the existence of a "dissipative anomaly", i.e. the presence of a non-vanishing scalar dissipation in the limit of small molecular diffusivity. Intermittency in the latter case has a natural Lagrangian interpretation connected to the existence of particles which separate even if starting in coinciding points 3, 4]. Such a mechanism is absent in inverse cascade, pointing toward the conclusion that inverse cascade regimes cannot be intermittent. Here we intend to investigate a similar issue on a purely Eulerian base.

Intermittent fluctuations are usually related to the scaling properties of the Navier Stokes equations [1]. Let us denote by $\boldsymbol{v}(\boldsymbol{x}, t)$ the velocity field of a turbulent flow satisfying the Navier Stokes equations:

$$
\partial_{t} \boldsymbol{v}+\boldsymbol{v} \bullet \nabla \boldsymbol{v}=-\nabla \pi+\nu \Delta \boldsymbol{v}+\boldsymbol{f}
$$

where $\pi=p / \rho$ ( $p$ being the pressure and $\rho$ the density), $\nu$ is the kinematic viscosity of the flow and $f$ is the (large scale) external forcing. Equations (1) show the remarkable property to be invariant under the scale transformation

$$
\boldsymbol{x} \rightarrow \lambda \boldsymbol{x}, \quad \boldsymbol{v} \rightarrow \lambda^{h} \boldsymbol{v}, \quad t \rightarrow \lambda^{1-h} t, \quad \nu \rightarrow \lambda^{1+h} \nu .
$$

Note that (2) implies for the energy dissipation: $\epsilon \rightarrow$ $\lambda^{3 h-1} \epsilon$, i.e. $\epsilon$ is constant if $h=1 / 3$ as predicted by the K41 theory. In the multifractal theory of turbulent flows, $h$ is supposed to be a fluctuating quantity, although on the average the rate of energy dissipation is constant [1]. The above scenario is complemented by the assumption that the statistical fluctuations are described by a scaling invariant probability distribution $P_{h} \sim \lambda^{3-D(h)}$ where $D(h)$ can be interpreted as the fractal dimension related to the fluctuations $\lambda^{h}$. Regardless of the geometrical interpretation of $D(h)$, the basic physical question is which is the mechanism determining the fluctuations of $h$ and why there are fluctuations in the energy flux. The above, scale invariant, scenario of the non equilibrium statistical properties of turbulent flows suggests a simple although not trivial picture of intermittency, related to the energy cascade mechanism. At scale $r$, the amount of kinetic energy due to turbulent fluctuations can be estimated as $\left(\delta_{r} v\right)^{2}$, where $\delta_{r} v=v(x+r)-v(x)$, and where we neglect vectorial indexes for simplicity. We expect that the rate of energy flux at scale $r$, denoted by $\epsilon_{r}$, is of the order of $\left(\delta_{r} v\right)^{2} / \tau_{r}$. Because energy transfer is due to non linear interactions, $\tau_{r}$ can be estimated as $r / \delta_{r} v$. Thus we obtain $\left(\delta_{r} v\right)^{3} \sim r \epsilon(r)$ which, as expected, is invariant under (2). As a result, the energy transfer statistics is strongly correlated to the fluctuations on the energy contents at different scales, $\left(\delta_{r} v\right)^{2}$, and to their dynamical properties, $\tau_{r}$. Moreover, the presence of strong intermittent fluctuations in the energy contents at different scales, reflects, via the equation of motions, into non-trivial fluctuations of the local eddy turn over times. Indeed, previous theoretical and numerical works have demonstrated that spatial and temporal properties of the energy cascade mechanism are strongly correlated [5, 6] .

The previous phenomenological arguments suggest that intermittency can be observed only if energy is flowing towards faster scales of motion. It is therefore tempting to argue that by decreasing the scaling exponents of the eddy turnover times along the cascade, 
i.e. by slowing down the energy transfer mechanism for fixed energy flux, intermittency should decrease and eventually disappear. Our aim in this letter is to provide clear evidence that the above conjecture holds.

Our analysis will be performed in the framework of shell models of turbulence (see [1, 7, 8] and references therein). The motivation to use shell models as a possible surrogate of the Navier-Stokes dynamics is twofold. First, shell models proved to be very successful in reproducing many of the statistical features of both 2 and 3 dimensional turbulent flows, being at the same time much easier to simulate numerically. Second, they are flexible enough to allow a structural change in their equations of motion which will allow us to directly probe the importance of time dynamics in fixing the intermittent properties of the energy transfer process (see below).

In a shell model, the basic variable describing the 'velocity field' at scale $r_{n}=\Lambda^{-n} r_{0} \equiv k_{n}^{-1}$, is a complex number $u_{n}$ satisfying a suitable set of non linear equations. There are many version of shell models which have been introduced in literature (see [8] for a recent review). Here we choose the one proposed in $[9]$ which is an improved version of the so-called GOY model [10, 11]

$$
\begin{aligned}
& \frac{d u_{n}}{d t}=i k_{n}\left[\Lambda u_{n+1}^{*} u_{n+2}+b u_{n-1}^{*} u_{n+1}-c \Lambda^{-1} u_{n-2} u_{n-1}\right] \\
& -\nu k_{n}^{2} u_{n}+f_{n}
\end{aligned}
$$

where $\Lambda=2, c=-(1+b)$ and $f_{n}$ is an external forcing. In shell models, we can associate $\delta_{r} v$ to $u_{n}$. Clearly equation (3) satisfies the scaling (2). The important point on shell models like (3) is that the statistical properties of intermittent fluctuations, computed either using $u_{n}$ or the instantaneous rate of energy dissipation, are in close qualitative and quantitative agreement with those measured in laboratory experiments, for homogeneous and isotropic turbulence. Thus, shell models provide an useful tool to investigate in a simple way the physical consequences of scaling (2) and intermittency. Moreover, at variance from $3 d$ Navier-Stokes equations, the computational complexity grows with Reynolds only as $R e^{1 / 2}$, allowing for reliable numerical studies also at very high Reynolds numbers.

It is easy to realize that also in shell models, $\tau\left(k_{n}\right)$ goes as $k_{n}^{h-1}$ as predicted by (2), i.e. the characteristic time for energy transfer decreases quite fast as the scale $k_{n}^{-1}$ is decreasing. If $\tau\left(k_{n}\right)$ is independent of $k_{n}$, the constantflux argument would suggest energy equipartition among all scales, i.e. $\left(\delta_{r} v\right)^{2} \sim \epsilon_{r}$. To be more specific, by constraining ourselves on shell models, let us modify (3) in such a way that there still exists an average Reynolds independent rate of energy dissipation $\epsilon=\left\langle\nu \sum_{n} k_{n}^{2}\left|u_{n}\right|^{2}\right\rangle$ while the statistical properties are invariant under the scaling transformation:

$$
k_{n} \rightarrow \lambda^{-1} k_{n}, \quad u_{n} \rightarrow \lambda^{h} u_{n}, \quad t \rightarrow \lambda^{x-h} t, \quad \nu \rightarrow \lambda^{1+h} \nu
$$

where $x$ is a fixed parameter in the equations. Note that (44) to hold, we should require that the non linear terms scale as $\lambda^{2 h-x}$. Note also that $\epsilon \sim \nu(\nabla v)^{2} \sim \lambda^{3 h-1}$, thus we do not change the constrain on the energy flux.

In order to satisfy the scaling (4), we introduce the following equations:

$$
\begin{aligned}
& \frac{d u_{n}}{d t}=i k_{0}^{1-x} k_{n}^{x}\left[u_{n+1}^{*} u_{n+2}+b u_{n-1}^{*} u_{n+1}\right. \\
& \left.+\frac{1+b}{2} u_{n-2} u_{n-1}\right]-\nu k_{n}^{1+x} k_{0}^{1-x} u_{n}+f_{n}
\end{aligned}
$$

where $k_{0}$ is the largest scale in the system. Clearly, we obtain the old shell model for $x=1$, while a direct inspection of (5) shows that (4) is satisfied for any $x$. Moreover, for $\nu=0$, the generalized energy, $Q=k_{0}^{x-1} \Sigma_{n} k_{n}^{1-x}\left|u_{n}\right|^{2}$, is conserved by non liner interactions. Thus, in a statistically stationary regime we have the 'energy' budget:

$$
\frac{1}{2} \frac{d Q}{d t}=0=P-\nu \sum_{n} k_{n}^{2}\left|u_{n}\right|^{2}
$$

where $P \propto \operatorname{Real}\left(\left\langle\sum_{n} k_{n}^{1-x} u_{n}^{*} f_{n}\right\rangle\right)$ is the energy input. Note that in this shell model energy fluctuations at scale $k_{n}$ are of the order $Q_{n}=k_{0}^{x-1} k_{n}^{1-x}\left|u_{n}\right|^{2}$ while the eddyturn-over time for the energy transfer is of the order

$$
\tau\left(k_{n}\right) \equiv 1 /\left(k_{0}^{x-1} k_{n}^{x} u_{n}\right) .
$$

Thus the energy flux through wavenumber $k_{n}$ can be proved rigorously to be of the order of $\Pi_{n}=k_{n}\left|u_{n}\right|^{3}$ as for the original shell model. Therefore, for all $x$, by keeping $\left\langle\Pi_{n}\right\rangle=$ const. we can achieve a constant energy flux:

$$
\left\langle\left|u_{n}\right|^{3}\right\rangle \sim k_{n}^{-1} .
$$

In summary, while for all $x$ the shell model (5) produces the same average energy flux, its dynamics is characterized by a different time-scaling properties which changes the eddy-turn-over hierarchy in the inertial range. In particular for small $x$, say $x \sim 1 / 3$, by combining (6) and (17) we should expect all eddy turn over times to be of the same order in the inertial range and therefore the energy to reach a quasi-equipartion state for fixed value of $\epsilon$. The shell model provides us the interesting possibility to study intermittency as function of $x$, i.e. as a function of the dynamical scaling (4) and (6). Intuitively, one can imagine that decreasing $x$ from its Navier-Stokes value $x=1$ induces a smoother and smoother energy transfer process towards smaller scales, fluctuations among different scales tend to equilibrate each other and, consequently, non-Gaussian fluctuations are depleted. Thus, as a function of $x$ the shell model (5) should exhibit a kind of phase transition from 'strong' intermittent fluctuations at $x=1$ to Gaussian non intermittent fluctuations at $x$ small. In this paper we support the above conjecture by numerical simulations of the model (5). Moreover, we will give a theoretical argument to estimate the value of $x_{c}$ below which intermittency starts to be depleted.

In order to keep $\epsilon$ constant, regardless of the value of $\nu$ and $x$, we use the forcing $f_{n}=A_{n} / u_{n}^{*}$ for $n=1,2$ 
and $f_{n}=0$ for $n>2$. We have performed a series of numerical simulations for different values of $x$, keeping the parameter $b=-0.4$ fixed. As a measure of intermittency, we compute the generalized kurtosis $G_{2 p}\left(k_{n}\right)=\left\langle\left|u_{n}\right|^{2 p}\right\rangle /\left\langle\left|u_{n}\right|^{2}\right\rangle^{p}$. Figure (10) shows the value of $G_{4}\left(k_{n}\right)$ for different values of $k_{n}$ as a function of $x$, while in the inset we show the value of $G_{6}\left(k_{n}\right)$. As one clearly see, for $x$ smaller than 0.5 a sharp decrease of $G_{4}$ and $G_{6}$ is observed. For small values of $x$ both $G_{4}$ and $G_{6}$ become equal to their Gaussian value. The above

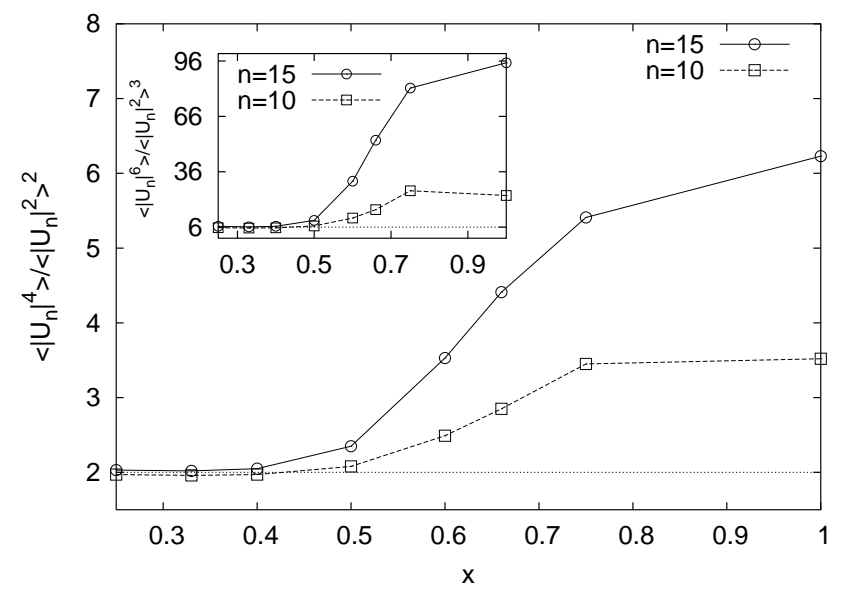

FIG. 1: The value of $G_{2 p}\left(k_{n}\right)=\left\langle\left|u_{n}\right|^{2 p}\right\rangle /\left\langle\left|u_{n}\right|^{2}\right\rangle^{p}$ for $p=2$ and $p=3$ (in the inset) as function of $x$ and for two different values of $k_{n}$, namely $n=10$ and $n=15$. The predicted value for the Gaussian Statistics (horizontal dotted line) is reported for comparison.

results tell us that intermittency is depleted for small $x$ in agreement with our intuitive argument. Also, within the numerical error bars, for $x>x_{c} \sim 0.5$, intermittent fluctuations seem to weakly depend on $x$.

We want now to understand why the transition to nointermittency fluctuations is observed for relatively large value of $x$, namely for $x \sim 0.4$. As we shall see in the following, $x_{c}=1 / 3+\Delta x$ where $\Delta x$ is due to intermittency correction. The argument goes as follows. Let us consider two scales $k_{n}$ and $k_{n+m}$. The corresponding times for the energy transfer are $\tau\left(k_{n}\right) \sim k_{n}^{h}$ and $\tau\left(k_{n+m}\right) \sim k_{n+m}^{h^{\prime}}$, where $h$ and $h^{\prime}$ are the values of the 'local' fluctuations of $u_{n} \sim k_{n}^{-h}$ and $u_{n+m} \sim k_{n+m}^{-h^{\prime}}$ respectively. For $x=1$ the probability that the ratio $\chi_{m}=\tau\left(k_{n+m}\right) / \tau\left(k_{n}\right)$ is larger than 1 is extremely small, already for $m=1,2$. When $x$ becomes smaller than 1 the probability $P\left(\chi_{m}>1\right)$ start growing, even for small $m$. If $\chi_{m}$ is much larger than 1 , then the energy transfer from scale $k_{n}$ to scale $k_{n+m}$ is stopped and energy tends to equipartition. In order to estimate $x_{c}$, let us compare, for a given scale separation $k_{m}$, the ratio between two eddy turn over times $T_{m}(q)=\left\langle\left(\chi_{m}\right)^{q}\right\rangle^{1 / m}$ :

$$
T_{m}(q) \sim\left(\int d h k_{m}^{(q h-q x-(3-D(h))}\right)^{1 / m} \sim k_{m}^{\frac{(-q x-\zeta(-q))}{m}}
$$

$\zeta(q)=i n f_{h}[q h+3-D(h)]$

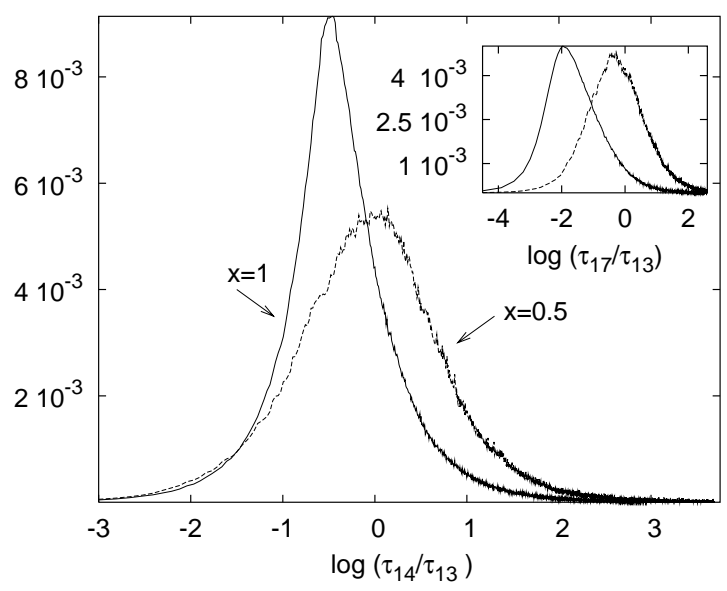

FIG. 2: Probability distribution of $\log \left(\tau\left(k_{14}\right) / \tau\left(k_{13}\right)\right)$ for two different values of $x$, namely $x=1$ and $x=0.5$. The theory presented in the paper predicts that intermittency is depleted at $x=0.5$ because $\tau\left(k_{n+1}\right) \sim \tau\left(k_{n}\right)$. In the inset we show the probability distribution of $\log \left(\tau\left(k_{17}\right) / \tau\left(k_{13}\right)\right)$

The above expression tells us that $T_{m}(q)>1$ for $x<x_{c}(q)=-\zeta(-q) / q$. Let us note that, because of convexity properties of $\zeta(q), x_{c}(q)$ is an increasing function of $q$. Thus, there is not a single value of $x$ below which intermittency is depleted, rather the transition to equipartition is continuous, different moments of the eddy turn over ratios behave in slightly different way. As a simple guide line the transition region is $\left[x_{c}(1), x_{c}(2)\right]=[0.4,0.42]$, estimated by using the $D(h)$ curve which fits the $\zeta(q)$ exponents as given by the She-Leveque formula [12]. A direct numerical in-

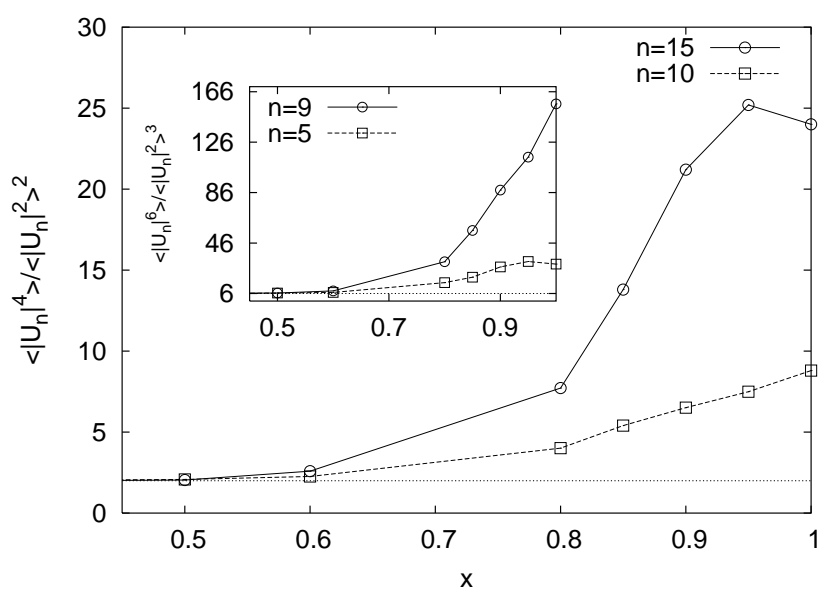

FIG. 3: Same quantities as in figure (1) for the model with parameter $b=-0.8$. The increase of intermittency at $x=$ 1 increases the critical value of $x$ for which thermodynamic equilibrium is attained.

vestigation gives further support to our previous argu- 
ment. In figure (2) we show the probability density function of the ratio $\tau\left(k_{14}\right) / \tau\left(k_{13}\right), \tau\left(k_{n}\right)$ being the instantaneous eddy turnover time at shell $k_{n}$. For $x=1$, we expect that $\tau\left(k_{14}\right)<\tau\left(k_{13}\right)$ with probability close to 1 , while, according to our estimate, we expect that for $x=0.5 \tau\left(k_{14}\right) \sim \tau\left(k_{13}\right)$ and intermittency is depleted. This is clearly shown in figure (2), where in the insert we also show the probability density function of $\log \left(\tau\left(k_{17}\right) / \tau\left(k_{13}\right)\right)$. Hence, we can conclude that for all scales $k_{n}$ the eddy turnover times become almost equal producing a kind of thermodynamic equilibrium in the generalized energy.

As a further check of our argument, we consider the shell model (5) for $b=-0.8$. In this case, the model at $x=1$ shows larger intermittent correction with respect to the case $b=-0.4$ previously considered. According to our argument for the transition to occur, we should expect that for $b=-0.8$, depletion of intermittency takes place for larger values of $x$, which is indeed the case as shown in figure (3).

In summary, we have presented the following main results: (i) we introduced a new version of the shell model which satisfies a generalization of the dynamical scaling (4); (ii) we have proposed a simple, although non trivial, argument for understanding how intermittency can depend on the scaling properties of the eddy turnover time; (iii) we have shown, by numerical simulations, that our argument is correct; (iv) we have provided a multifractal estimate of the critical value, $x_{c}$ where intermittency should disappear.

We thank F. Toschi for discussion in a early stage of this work.
[1] U. Frisch, Turbulence: The legacy of A.N. Kolmogorov (Cambridge University Press, Cambridge, 1995).

[2] G. Boffetta, A. Celani, and M. Vergassola, Phys. Rev. E 61, R29 (2000).

[3] G. Falkovich, K. Gawedzki and M. Vergassola Rev. Mod. Phys. 73913 (2001).

[4] A. Celani, M. Cencini, A. Mazzino and M. Vergassola New J. Phys. 6, 72 (2004).

[5] V.S. L'vov, E. Podivilov and I. Procaccia. Phys. Rev. E 557030 (1997).

[6] L. Biferale, G. Boffetta, A. Celani and F. Toschi. Physica D 127187 (1999).
[7] T. Bohr, M. H. Jensen, G. Paladin, and A. Vulpiani, Dynamical Systems Approach to Turbulence (Cambridge University Press, Cambridge, 1998).

[8] L. Biferale, Ann. Rev. Fluid. Mech. 35, 441 (2003).

[9] V.S. L'vov, E. Podivilov, A. Pomyalov, I. Procaccia, and D. Vandembroucq, Phys. Rev. E 58, 1811 (1998).

[10] M. Yamada and K. Ohkitani, Phys. Rev. Lett. 60, 983 (1988).

[11] M.H. Jensen, G. Paladin and A. Vulpiani, Phys. Rev. A. 43798 (1991).

[12] Z.S. She and E. Leveque, Phys. Rev. Lett. 72336 (1994). 